\title{
Status Epilepticus in Children: A Study of 54 Cases
}

\author{
Fuyong Jiao', Lei Ma², Robert Ouvrier³, Patrick Ho³, Alhaji Adam Abubakari ${ }^{4,5,6,7^{*}}$ \\ ${ }^{1}$ Children's Hospital of Shaanxi Provincial People's Hospital, Xi'an, China \\ ${ }^{2}$ Xi'an Medical College, Xi'an, China \\ ${ }^{3}$ Royal Alexandra Hospital for Children, Sydney, Australia \\ ${ }^{4}$ Tamale Teaching Hospital, Tamale, Ghana \\ ${ }^{5}$ School of Medicine and Health Sciences, University for Development Studies, Tamale, Ghana \\ ${ }^{6}$ Shenyang Medical College, Shenyang, China \\ ${ }^{7}$ Feng Tian Hospital affiliated to Shenyang Medical College, Shenyang, China \\ Email: ^biemaba1@gmail.com
}

How to cite this paper: Jiao, F.Y., Ma, L., Ouvrier, R., Ho, P. and Abubakari, A.A. (2018) Status Epilepticus in Children: A Study of 54 Cases. Open Journal of Emergency Medicine, 6, 88-98.

https://doi.org/10.4236/ojem.2018.64011

Received: October 20, 2018

Accepted: November 27, 2018

Published: November 30, 2018

Copyright $\odot 2018$ by author(s) and Scientific Research Publishing Inc. This work is licensed under the Creative Commons Attribution International License (CC BY 4.0).

http://creativecommons.org/licenses/by/4.0/

\begin{abstract}
Objectives: To review the clinical character, the management and outcome of status epilepticus in children. Methodology: We conducted a retrospective review of 54 cases treated between 1996 and 1997 at the Royal Alexandra Hospital for Children, Sydney, Australia. Among the variables collected for analysis in this study were age, sex, etiology of status epilepticus, median length of Paediatric Intensive Care Unit (PICU) stay and days of mechanical ventilation, and mortality of status epilepticus. Results: Of the patients with status epilepticus, $61 \%$ were male and $39 \%$ female, with an age range of 3 months to 15 years (mean 5.3 years). The etiology of status epilepticus is largely age related, with acute causes common in $1-3$ year and $4-7$ year ages, 44 cases $(81.5 \%)$. The etiology of status epilepticus included febrile (35.18\%), acute symptomatic (27.58\%) and idiopathic (16.6\%), total was 44 cases (81.4\%). Median length of PICU stay and days of mechanical ventilation were $3.02 \pm 1.6$ days and $1.24 \pm 0.5$ days respectively. Mortality was $5.3 \%$. Most patients were treated with diazepam and phenytoin. The total number of the patients with a favorable outcome was $41(75.89 \%)$. Conclusions: One of the most common neurologic emergencies in children of status epilepticus remains a major problem in morbidity and mortality. There are multiple causes of status epilepticus that include the categories of idiopathic, febrile, acute symptomatic and remote symptomatic. Intravenously administered phenytoin and diazepam remains the first-line therapy for status epilepticus. Most of the patients will respond to this treatment. The etiology of status epilepticus included febrile (35.18\%), acute symptomatic (27.58\%), idiopathic
\end{abstract}


(16.6\%) and total was $44(81.4 \%)$.

\section{Keywords}

Status Epilepticus, Children, Management, Idiopathic, Mortality, Paediatriac Intensive Care Unit (PICU)

\section{Introduction}

Status epilepticus (SE) is one of the most common emergencies in paediatric neurology and is associated with a high mortality and morbidity, with an incidence of 20 per 100,000 children per year [1] [2]. It is more frequent in children than in adults and occurs in a variety of settings, especially in children with infections, and patients with previously established epilepsy, cerebral malformations, hypoxia, hypoglycaemia and head trauma. In many cases, SE can be the first unprovoked manifestation of a seizure disorder. Mortality rates associated with SE in children between 3 and 20 percent have been reported, with higher rates noted if a significant metabolic disturbance is present or a delay in termination [3] [4]. Although many studies have been devoted to the clinical, Electro Encephalography (EEG) and neuropathological features of SE, however, little is known on etiological and prognostic features of SE. This paper therefore presents the etiological, clinical characteristics, effect of treatment and prognosis in a large group of children with status epilepticus, with special attention been paid to their intensive care course and the presence of anticonvulsant drug toxicity.

\section{Materials and Methods}

The study was approved by the ethics committee of the Royal Alexandra Hospital for Children, Westmead, Sydney, Australia. The international classification of disease (ICD-9-CM) codes for seizures (780.3), SE (345.3) and convulsions (345.9) were used to classify patients (Dreifuss et al., 1981). Sixty two (62) patients presenting with SE hospitalized from 1, January 1996 to 31, December 1997 (with 88 admissions) were identifie, however, only 56 patients (with 61 admissions) had records available during the time of research (Dreifuss et al., 1981).

Status Epilepticus was defined as any continuous seizure lasting longer than 30 minutes or a series of seizures without return of consciousness for at least 30 minutes. Seizures were classified as generalized (absence, myoclonic, clonic, tonic, tonic-clonic, atonic) or partial (simple, complex, secondarily generalized) according to the classification of the International League against Epilepsy [5]. The etiology of SE was identified using the modification of the classification of Hasue et al. [4] [6] as follows:

1) Idiopathic: no acute CNS or metabolic dysfunction 
2) Febrile: status epilepticus provoked only by fever $\left(>38.4^{\circ} \mathrm{C}\right)$

3) Acute symptomatic: SE occurring during an acute illness with known CNS involvement (eg: meningitis)

4) Remote symptomatic: SE without acute causes occurring in patients with a prior history of CNS insult known to be associated with increased risk of convulsions

5) Progressive neurological occurring during progressive neuropathy (eg: neurocutaneous diseases)

Utilizing the advice definitions, 54 patients were selected, 47 patients had generalized convulsive status (grand mal), 2 patients had partial SE, 3 patients had non-convulsive SE and 2 patients had tonic status. All patients' diagnoses were confirmed by computarized tomography (CT), Magnetic Resonance Imaging (MRI), Electro Encephalography (EEG), cerebrospinal fluid findings and(or) culture, except 2 patients who suffered neurofibromatosis and neurocutaneous disease (Sturge-Weber syndrome) who were documented by biopsy. The 2 patients who died underwent autopsy. All patients received standard initial evaluation and therapy including physical examination, Creatine Phosphokinase (CPK), Serum Glutamic Oxaloacetic (SGOT), Complete Blood Count (CBC), phenytoin and phenobarbital serum levels, and other anticonvulsant levels as indicated.

Parameters established by the hospital's clinical laboratory were used as acriteria for identifying therapeutic and subtherapeutic drug ranges. The therapeutic range for phenytoin was $40-89 \mathrm{umol} / \mathrm{L}$, phenobarbital $60-120 \mathrm{umol} / \mathrm{L}$ and carbamazepine 15 - $40 \mathrm{umol} / \mathrm{L}$.

Status Epilepticus is a medical emergency requiring prompt therapy. In the hospital, the treatment protocol used for SE consisted of three steps, similar to what others recommend:

1) Benzodiazepine (BZD) and Phenytoin (PHT) to terminate and prevent seizure recurrence. Main BZD used were Diazepam (DZP) and Clonazepam (CZP). Diazepam's initial dose was $0.25-0.4 \mathrm{mg} / \mathrm{kg}(\max 10 \mathrm{mg}$ ), rate of $<2$ $\mathrm{mg} / \mathrm{min}$. The dose often needed to be repeated within 10 - 15 minutes (Max 40 mg over 24 hours). Some patients received rectal administration at an initial dose of $0.5 \mathrm{mg} / \mathrm{kg}$ ( $\max 20 \mathrm{mg}$ ). Rectal DZP is most useful in prehospital settings. Phenytoin: IV loading dose of $15-20 \mathrm{mg} /$ grate $<0.5-1.0 \mathrm{mg} / \mathrm{kg} / \mathrm{min}(<4$ years old) or $25 \mathrm{mg} / \mathrm{min}$ ( $>4$ years old). The combination of IV DZP and PHT stopped seizures in almost all the patients.

If this approach fails:

2) Phenobarbitone (PB) as the third drug is added, loading dose of $20 \mathrm{mg} / \mathrm{kg}$ (max $100 \mathrm{mg}$ ), rate $<100 \mathrm{mg} / \mathrm{min}$.

3) General anaesthesia (GA) is utilized if SE continues $>60$ minutes or the first and second line drugs fail. Short barbiturate anaesthesia: this is induced by a short-acting barbiturate-thiopentone sodium, administered by IV at $30 \mathrm{mg} / \mathrm{kg}$ over 1 hour, then $5 \mathrm{mg} / \mathrm{hr}$ as maintenance infusion, increased to $10-20$ $\mathrm{mg} / \mathrm{kg} / \mathrm{h}$. The patients were admitted to the PICU, intubated and adapted to and placed on assisted ventilation, continuous EEG and cardiorespiratory monitoring. Arterial and intravenous lines were placed in all patients. Anaesthesia was 
usually maintained at this rate for approximately 24 hours. If seizure discharges return as the general anaesthesia is slowly withdrawn, EEG should be re-converted to burst suppression or isoelectric tracing with GA during the following 24 hours.

\section{Results}

\subsection{Age and Sex}

Fifty four (54) cases with SE were identified. Of these, there were 33 males and 21 females. 24 (44.4\%) cases were less than 3 years of age and 20 (37.1\%) cases between 4 to 7 years of age. Together, they represented $44(81.5 \%)$ cases. The average age was 5.3 years, with an age range of 3 months to 15 years old (see Table 1).

\subsection{Etiology of Status Epilepticus}

Etiology of SE was idiopathic in 9 cases, febrile in 19 cases and acute symptomatic in 16 cases. The most common etiologies of SE were febrile, idiopathic and acute symptomatic (Table 2).

Table 1. Age and sex distribution of Status Epilepticus.

\begin{tabular}{|c|c|c|c|}
\hline \multirow{2}{*}{ Age } & \multicolumn{2}{|c|}{ Number of Cases } & \multirow{2}{*}{ Total } \\
\hline & Male & Female & \\
\hline $1 \mathrm{M}-3 \mathrm{M}$ & 2 & 1 & 3 \\
\hline $4 \mathrm{M}-1 \mathrm{Y}$ & 5 & 5 & 10 \\
\hline $2 Y-3 Y$ & 5 & 6 & 11 \\
\hline $4 Y-7 Y$ & 12 & 8 & 20 \\
\hline $8 \mathrm{Y}-15 \mathrm{Y}$ & 7 & 3 & 10 \\
\hline
\end{tabular}

Table 2. The etiology of Status Epilepticus.

\begin{tabular}{cc}
\hline The Etiology of Status Epilepticus & Number \\
\hline Idiopathic & $9(17 \%)$ \\
Febrile & $19(35 \%)$ \\
Acute Symptomatic & $16(30 \%)$ \\
CNS infection & $9(17 \%)$ \\
Brain Trauma & $3(6 \%)$ \\
Sepsis & $2(4 \%)$ \\
Hydrocephalus & $2(4 \%)$ \\
(shunt obstruction) & \\
Remote Symptomatic & 0 \\
Congenital CNS malformation & $3(6 \%)$ \\
Prior CNS insult & $3(6 \%)$ \\
Progressive Neurological & \\
Neurocutaneous & $2(4 \%)$ \\
Metabolic & $2(4 \%)$ \\
\hline
\end{tabular}




\subsection{Type of Seizure}

Grand mal was the commonest type and was seen in 47 cases (87.3\%). The remaining cases included partial SE with 2 cases, tonic status with 2 cases and non-convulsant SE with 3 cases. Previously diagnosed and treated epilepsy was present in $12(22.22 \%)$ cases.

\subsection{Drugs Used}

\subsubsection{Serum Anticonvulsant Level (Use of Phenytoin)}

Within the 61 admissions, intravenous phenytoin was employed in 29 (47.5\%) cases (maximum serum levels were known, ranging from 65 to $270 \mathrm{umol} / \mathrm{L}$, therapeutic range of $40-80 \mathrm{umol} / \mathrm{L}$ ).

Phenytoin was helpful in preventing PICU admission in 5 admissions, 3 of which belonged to the febrile category, 1 idiopathic and 1 progressive. One child was admitted to ICU without mechanical ventilation after phenytoin.

In one case the child was experiencing respiratory depression while on phenobarbitone. Phenytoin was substituted for phenobarbitone in this case because of its good seizure control and no obvious respiratory depression.

In 4 admissions, progressive ataxia, nystagmus or intention tremor were observed associated with phenytoin use. No cardiac complications were reported. These were the children with acute bacterial meningitis and ALL. No deaths were considered a consequence of phenytoin use. None of the children suffering from the side effects of phenytoin had been on prior treatment with phenytoin. Three levels available in the patients with side effects were 85,113 and 270 umol/l.

\subsubsection{Use of Phenobarbitone}

Intravenous phenobarbitone was used in 20 (33\%) admissions suffering Status Epilepticus. Of these patients, the etiologies included 5 febrile, 3 acute CNS, 7 idiopathic, 2 chronic CNS and 3 progressive. Ten (10) maximum serum levels were obtainable, ranging from 75 to $195 \mathrm{umol} / \mathrm{l}$ (therapeutic 40 - $130 \mathrm{umol} / \mathrm{l}$ ).

In 2 cases, PICU admission was not required for the management of Status Epilepticus. One child had febrile SE and the other had lissencephaly, clinically requiring the use of phenobarbitone. In all the other cases, phenobarbitone use was associated with mechanical ventilation.

In one case, irritability developed and was attributed to phenobarbitone. The child who expired with ALL also had phenobarbitone injection. Phenobarbitone levels were not known in either case.

\subsection{Median Length of PICU Stay and Days of Mechanical Ventilation}

Among children admitted to the PICU between January 1996 and December 1997, 45\% were patients with SE. 45 (83.3\%) of 54 cases with SE were admitted to the PICU. The median length of the PICU stay for the patients was $3.02 \pm 1.6$ days (range of 0.5 - 9 days), total was 136 days in the 45 cases. The mean days of 
mechanical ventilation for the patients was $1.24 \pm 0.5$ days (range of $9 \mathrm{~h}-10 \mathrm{~d}$ ), total was 55.8 days in all the cases (Table 3 ).

\subsection{Outcome}

Status Epilepticus (SE) is a serious problem, since it is often associated with high mortality if it persists longer than 60 minutes. Therefore, this medical emergency requires prompt therapies including a proper airway ensured, oxygen administered and anticonvulsant drugs used as soon as possible to terminate the seizure. The total number of the patients with a favorable outcome in this series was 41 . Poor response in 8 cases occurred mainly because of complications and inadequate dosages. In 6 refractory cases, thiopentone effected control seizure. Thirty six (36) patients had been administered diazepam before admission to the hospital. Phenytoin combined with diazepam was the first line anticonvulsant drugs.

The overall mortality was 3 (5.3\% of all the patients, $4.9 \%$ of all the admissions) in Table 4.

The deaths in the acute Central Nervous System (CNS) group were due to acute bacterial meningitis in one and encephalopathy associated with hepatic failure in the other. The death in the progressive encephalopathy group was in a child with relapsed acute lymphoblastic leukemia post bone marrow transplantation.

Table 3. Clinical care data.

\begin{tabular}{cc}
\hline Recurrent Seizure in PICU & $28(51.8 \%)$ \\
PICU Admission & $45(83.1 \%)$ \\
Intubation required & $41(75 \%)$ \\
Length of PICU (day) & $3.02 \pm 1.6$ (Range $0.5-9$ days) \\
Length of Mechanical ventilation (day) & $1.24 \pm 0.5$ days (Range $9 \mathrm{~h}-10 \mathrm{~d})$ \\
Total days of PICU of the patients & 58 \\
Total days of ventilation of the patients & 3 \\
Number of deaths & 6 \\
General anaesthesia-Thiopentone & \\
\hline
\end{tabular}

Table 4. Mortality rate of SE.

\begin{tabular}{cc}
\hline Etiology & Mortality 1\% Each Group \\
\hline Febrile & 0 \\
Acute CNS injury & $2(22 \%)$ \\
Idiopathic & 0 \\
Chronic CNS injury & 0 \\
Progressive encephalopathy & $1(11 \%)$ \\
\hline
\end{tabular}




\section{Discussion}

Despite significant advances in treatment, SE is still one of the common causes of mortality and neurologic sequelae in infants and children, particularly in those with chronic encephalopathies and pre-existing epilepsy [7] [8] [9] [10]. A revised operational definition based on the indication to commence treatment has defined SE as seizures of a duration of five minutes or more [11]. In the present study, patients 3 months to 3 years of age (24 cases, 44.4\%) and 4 to 7 years (20 cases, $37.1 \%$ ) represented $81.5 \%$ of the cases. The commonest age group of SE was $4 \mathrm{M}-1 \mathrm{Y}$ and $1 \mathrm{Y}-3 \mathrm{Y}$.

Our data showed that for the etiology of SE, half were idiopathic and febrile, 9 (16.6\%) and $19(35.18 \%)$ respectively, infection is also one of the major causes with a total of $9(16.66 \%)$ cases. This is different from Scholtes et al. (1994) who reported that chronic non-transgressive encephalopathy was the primary cause [8] [9] [12] [13]. The literature suggests that the etiology and outcome of SE in children are different from those in adult patients. Within etiologies, such as idiopathic, febrile, or remote symptomatic, there was no difference in outcome among the two groups.

There were 12 patients with pre-existing epilepsy and 7 patients had anticonvulsant drug withdrawal before the onset of status, so this may have played a role in inducing SE in a minority of patients [14].

Mortality rate in our study was 5.3\%. Another report indicated a rate of 6 to $7 \%$ (Stephen et al., 1989). It appears that the co-existing severe systemic illness in our patients led to relatively high mortality rate (i.e. Acute Lymphocytic Leukemia (ALL), Multiple Organ Failure (MOF) and fulminant liver failure). Mortality and morbidity for SE are associated with three major factors, namely damage to the CNS caused by acute insult, the systemic effects of repeated convulsions and neural injuries from repetitive electrical discharges within the CNS. The first factor is clearly associated with the etiology of SE and the other two factors are related to the duration of SE. A recent study of adults by Stecker showed similar conclusions as ours regarding outcome and mortality of SE [15]. Two of our study patients underwent post-mortem examination of the brain and body. $\mathrm{Pa}$ tient 1 was a 3 -month old girl. There was severe extensive cystic infarction of the white mater of the cerebral hemispheres with evidence of neuronal dropout on the cerebral cortices, cerebellar pukinje cells and dentate nucleus neurons. Evidence of recent hypoxic damage to neurons of thalamus was seen in patient 2 , a 16-month old girl. In this patient, the autopsy revealed changes of bacterial meningitis. There were large numbers of neutrophils in the subarachnoid space of both cerebral hemispheres and cerebellum. The inflammatory infiltration extended to the sulcus and there was patchy inflammatory infiltration of the cerebellar folia.

The most common complications of SE included pneumonia (4\%) and tonsillitis (5\%) and intracranial hypertension (5\%). The existence of more than one medical complication was related to worse outcome. The most severe complica- 
tions were respiratory insufficiency. In addition, cardiac arrhythmia, hypotension and phlebitis also contributed to a poor result. A study in India shows appropriate prehospital management and treatment targeting resolution of cardiovascular dysfunction during resuscitation could reduce mortality in children with SE [10] [16] [17]. Consequently, the importance of prompt treatment of medical complications during SE has been stressed.

In our study, SE was categorized by etiology, where known. The classification included febrile, acute symptomatic, idiopathic, remote symptomatic and progressive neurological categories (Table 2).

Most of the presentations of SE required PICU admission. Most of the Paediatrics Intensive Care Unit (PICU) admissions required mechanical ventilation probably reflecting good clinical judgement of the Emergency and PICU physicians in selecting admitted cases.

This study confirmed the findings of Maytal, et al. (1989) regarding mortality. Status epilepticus itself is associated with low mortality. It is the concurrent pathological processes (Acute CNS injury or progressive encephalopathy) that mostly determine the cause of death [7] [9] [10] [17].

There was great concern for the emergency or PICU physicians regarding the oversubscribing of benzodiazepines or barbiturates in the acute resuscitative period, hence the necessity for PICU admission. The use of phenytoin has been encouraged. Most emergency centers will recommend the use of intravenous phenytoin when the seizure persists for more than 30 minutes. According to this study, around $50 \%$ of the cases employed phenytoin for seizure control. In $21 \%$ $(6 / 29)$ of children receiving intravenous phenytoin, the need for intubation was avoided and in terms of budget, 5 PICU beds were saved. However, it could not be established whether the SE stopped spontaneously because of the direct effect of phenytoin. 13.8\% (4/29) of children receiving intravenous phenytoin suffered from acute cerebellar toxicity. Except for 1 child with a level of $270 \mathrm{umol} / \mathrm{l}$, there was no correlation of complications with the levels, nor the prior treatment with phenytoin. There were no other side effects seen in this study, although we did not follow any of these children up after discharge. With this relatively small study, it seems the use of phenytoin should be encouraged to prevent the need for assisted ventilation and its possible adverse effects.

Most of the phenobarbitone use was in the febrile or the idiopathic group. It prevented only 2 mechanical ventilations. This might reflect the sedative nature of the drug itself. Only 1 behavioral side effect was recorded.

We have demonstrated that intravenously administered diazepam and phenytoin remained the first-line therapy for SE [18]. Phenytoin combined with diazepam is effective and safe. Furthermore, there has been a lot of research on whether intravenous (IV) levetiracetam or IV phenytoin is the better second line treatment for the emergency management of CSE in children [13] [19] [20] [21], suggesting that IV levetiracetam is safer and more effective. More than half of the patients will response to the initial treatment. Refractory cases of SE may necessitate treatment with high-dose phenobarbital or inhalation of anaesthetic 
agents. Proper airway must be ensured, oxygen administered, blood pressure supported and intracranial pressure controlled in a timely manner.

One of the most recent major advances in management of seizure disorders is availability of methods for rapid determination of anticonvulsant blood levels. Dosage schedule of anticonvulsant can now be adjusted to maintain serum drug levels within the therapeutic ranges. This provides a means of checking patient compliance and provides insights of drug metabolism of these compounds in individual patients. This is particularly important in young infants who may have variable absorption, when seizures are uncontrolled on therapy, drug toxicity is suspected or changes in drug treatment are planned. Diazepam is a good agent to arrest SE, but should not be used in combination with phenobarbital because of the risk of hypotension and respiratory depression, particularly in infants [13] [17] [21].

This study is limited by a small sample size of 54 persons, therefore, further study should be carried out on a larger sample size. Also, this study was conducted in a short term, hence further studies should be carried out in a long term $>1$ year.

The study therefore reviewed the clinical character, the management and outcome of status epilepticus in children.

\section{Acknowledgements}

The authors would like to acknowledge the support of the New Children's Hospital for the study in the two hospitals.

\section{Conflicts of Interest}

The authors declare no conflicts of interest regarding the publication of this paper.

\section{References}

[1] Chin, R.F., Neville, B.G., Peckham, C., et al. (2006) Incidence, Cause, and Short-Term Outcome of Convulsive Status Epilepticus in Childhood: Prospective Population-Based Study. The Lancet, 368, 222-229. https://doi.org/10.1016/S0140-6736(06)69043-0

[2] Novorol, C.L., Chin, R.F.M. and Scott, R.C. (2007) Outcome of Convulsive Status Epilepticus: A Review. Archives of Disease in Childhood, 92, 948-951. https://doi.org/10.1136/adc.2006.107516

[3] Frisby, J.R. (1990) Status Epilepsy. In: On T.E., Ed., Intensive Care Manual, Butterworths, Sydney, Australia, 266-269.

[4] Maytal, J., Chinnar, S., Moshe, S.L., et al. (1989) Low Morbidity and Mortality of Status Epilepticus in Children. Pediatrics, 83, 323-331.

[5] Dreifuss, F.E., Bancaud, J. and Henriksen, O., Commission on Classification and Terminology of the International League against Epilepsy (1981) Proposal for Revised Clinical and Electroencephalographic Classification of Epileptic Seizures. Epilepsia, 22, 489-501. https://doi.org/10.1111/j.1528-1157.1981.tb06159.x

[6] Hauser, W.A., Anderson, V.E., Loewenson, R.B. and McRoberts, S.M. (1982) Sei- 
zure Recurrence after a First Unprovoked Seizure. New England Journal of Medicine, 307, 522-528. https://doi.org/10.1056/NEJM198208263070903

[7] Phillips, S.A. and Shanahan, R.J. (1989) Etiology and Mortality of Status Epilepticus in Children: A Recent Update. Archives of Neurology, 46, 74-76.

https://doi.org/10.1001/archneur.1989.00520370076023

[8] Jiao, F.Y., Guo, X.Y., Lin, J. and Cui, W. (2010) A Randomized Trial of Ligustrazini Hydrochlorioi in the Treatment of Viral Encephalitis in Children. Journal of Nepal Pediatric Society, 30, 119-122. https://doi.org/10.3126/jnps.v30i2.3451

[9] Jiao, F.Y., Zhang, X.Y., Bai, T.M., Lin, J., Cui, W. and Liu, B.W. (2011) Clinical Evaluation of the Function of Hypothalamo-Pituitary-Thyroid Axis in Children with Central Nervous System Infections. Italian Journal of Pediatrics, 37, 11. https://doi.org/10.1186/1824-7288-37-11

[10] Jiao, F.Y., Cao, H.C., Liu, Z.Y., Wu, S. and Wong, H.B. (1992) The Use of Blood Glucose/CSF Glucose Ratio in the Diagnosis of CNS Infection in Infants and Children. Journal of the Singapore Pediatric Society, 34, 191-198.

[11] Lowenstein, D.H., Bleck, T. and Macdonald, R.L. (1999) It's Time to Revise the Definition of Status Epilepticus. Epilepsia, 40, 120-122. https://doi.org/10.1111/j.1528-1157.1999.tb02000.x

[12] Scholtes, F.B., Renier, W.O. and Meinardi, H. (1994) Generalized Convulsive Status Epilepticus: Causes, Therapy, and Outcome in 346 Patients. Epilepsia, 35, 1104-1112. https://doi.org/10.1111/j.1528-1157.1994.tb02562.x

[13] Jiao, F.Y., et al. (1997) Randomized, Controlled Trial of High-Dose Intravenous Pyridoxine in the Treatment of Recurrent Seizures in Children. Pediatric Neurology, 17, 54-57. https://doi.org/10.1016/S0887-8994(97)00035-0

[14] Maytal, J., Novak, G., Ascher, C., et al. (1996) Status Epilepticus in Children with Epilepsy: The Role of Antiepileptic Drug Levels in Prevention. Pediatrics, 98, 1119-1121.

[15] Stecker, M.M., Kramer, T.H., Raps, E.C., et al. (1998) Treatment of Refractory Status Epilepticus with Propofol: Clinical and Pharmacokinetic Findings. Epilepsia, 39, 18-26. https://doi.org/10.1111/j.1528-1157.1998.tb01269.x

[16] Santhanam, I., Yoganathan, S., Sivakumar, V.A., et al. (2017) Predictors of Outcome in Children with Status Epilepticus during Resuscitation in Pediatric Emergency Department: A Retrospective Observational Study. Annals of Indian Academy of Neurology, 20, 142-148. https://doi.org/10.4103/aian.AIAN_369_16

[17] Jiao, F.Y., Guo, R.L. and Ma, F.R. (1986) Differential Diagnosis of Central Nervous System Infections by Alkaline Phosphatase in the CSF. New Zealand Medical Journal, 99, 124.

[18] Cascino, G.D. (1996) Generalized Convulsive Status Epilepticus. Mayo Clinic Proceedings, 71, 787-792. https://doi.org/10.1016/S0025-6196(11)64844-1

[19] Dalziel, S.R., Furyk, J. and Bonisch, M. (2017) Amulticentre Randomised Controlled trial of Levetiracetam versus Phenytoin for Convulsive Status Epilepticus in Children (Protocol): Convulsive Status Epilepticus Paediatric Trial (ConSEPT)-A PREDICT Study. BMC Pediatrics, 17, 152. https://doi.org/10.1186/s12887-017-0887-8

[20] Lyttle, M.D., Gamble, C. and Messahel, S. (2017) Emergency Treatment with Levetiracetam or Phenytoin in Status Epilepticus in Children-The EcLiPSE Study: Study Protocol for a Randomised Controlled Trial. Trials, 18, 283. https://doi.org/10.1186/s13063-017-2010-8 
[21] Jiao, F.Y., Wang, J.Y., Zhang, X.Y. and Mu, Z.L. (2016) The Situation of Suppression Seizures with Diazepam by Rectal Administration in China. Journal of Pediatrics and Palliative Care, 1, 9-12. 